\title{
SUPLEMENTACIÓN POSTDESTETE DE CORDERAS PELIBUEY $x$ BLACKBELLY EN PASTOREO EN EL TRÓPICO HÚMEDO
}

\author{
SUPPLEMENTAL FEED DURING POSTWEANING GROWTH OF \\ PELIBUEY x BLACKBELLY EWE LAMBS UNDER GRAZING IN THE HUMID TROPIC
}

Cadenas, J.A. ${ }^{1}$, Oliva-Hernández, J. ${ }^{1,2}$, Hinojosa, J.A. ${ }^{2}$ y Torres-Hernández, G. ${ }^{3}$

\author{
${ }^{1}$ Universidad Popular de la Chontalpa. Tabasco. México. \\ ${ }^{2}$ Campo Experimental Huimanguillo. Instituto Nacional de Investigaciones Forestales, Agrícolas y Pecuarias \\ Tabasco. México. olivajh20@yahoo.com.mx \\ ${ }^{3}$ Colegio de Postgraduados. Campus Montecillo. Estado de México. México.
}

\section{Palabras clave adicionales}

Ganancia de peso. Ovino de pelo. Comportamiento productivo postdestete.

\section{RESUMEN}

El objetivo de este estudio fue evaluar el efecto del complemento alimenticio en el comportamiento productivo postdestete de corderas Pelibuey $x$ Blackbelly manejadas bajo condiciones de pastoreo. Veintiocho corderas Pelibuey x Blackbelly se asignaron a uno de dos tratamientos: 1) complementación alimenticia baja: 0,18 Mcal de energía metabolizable (EM) cordera-1 $\mathrm{d}^{-1}$ y $10,5 \mathrm{~g}$ de proteína bruta (PB) cordera ${ }^{-1} \mathrm{~d}^{-1} \circ 2$ ) complementación alimenticia media: 0,54 Mcal de EM y $31,6 \mathrm{~g}$ de PB cordera $^{-1} \mathrm{~d}^{-1}$, durante 70 días. La complementación alimenticia no afectó $(p>0,05)$ al peso corporal y la ganancia diaria de peso (GDP). Se concluye, que en corderas Pelibuey $x$ Blackbelly en pastoreo, la complementación alimenticia media no mejora la GDP con respecto a la complementación alimenticia baja.

\section{SUMMARY}

The objective of this study was to evaluate the effect of supplemental feed on postweaning performance of Pelibuey x Blackbelly ewe lambs managed under grazing conditions. Twenty eight Pelibuey x Blackbelly female lambs were assigned to one of two treatments: 1) low feed supplementation: $0.18 \mathrm{Mcal}$ of metabolizable energy (EM) lamb-1 $\mathrm{d}^{-1}$ and $10.5 \mathrm{~g}$ of crude protein (PB) lamb ${ }^{-1} \mathrm{~d}^{-1}$ and 2) medium feed supplementation: $0.54 \mathrm{Mcal}$ of EM and $31.6 \mathrm{~g}$ of PB lamb $^{-1} \mathrm{~d}^{-1}$,

Recibido: 30-4-08. Aceptado: 12-5-08.

\section{AdDitionAL KEYWORDS}

Body weight gain. Hair sheep. Postweaning performance.

offered during 70 days. The supplement feed did not affect $(p>0.05)$ body weight and average daily body weight gain (GDP). It is concluded that in grazing Pelibuey $x$ Blackbelly ewe lambs the medium feed supplementation did not improve the daily body weight gain with respect to a low feed supplementation.

\section{INTRODUCCIÓN}

En la región tropical de México, el manejo agronómico de la pradera no es suficiente para que el cordero de razas de pelo, optimice su potencial de crecimiento durante la etapa postdestete, debido a que las demandas de energía y proteína, no son cubiertas con facilidad a través del consumo voluntario de gramíneas tropicales, por lo que resulta conveniente, en estos casos, proporcionarles un complemento alimenticio que permita que el cordero exprese su potencial productivo (Oliva y Vidal, 2001. Con base en lo planteado anteriormente, el objetivo del estudio consistió en determinar la influencia del nivel de complementación energética y proteínica en el peso corporal y la ganancia de peso diaria (GDP) de las corderas Pelibuey x Blackbelly en pastoreo. 


\section{MATERIAL Y MÉTODOS}

El experimento se realizó en una fínca ovina localizada en Huimanguillo, Tabasco, México ( $\left.17^{\circ} 50^{\prime} \mathrm{LN}, 9^{\circ} 23^{\prime} \mathrm{LO}\right)$, a una altitud de $20 \mathrm{msnm}$. El clima de la región es cálido con lluvias todo el año (Af) y la temperatura media anual de $27,8^{\circ} \mathrm{C}$ (Anónimo, 2007).

El ensayo tuvo una duración de 81 días, y se dividió en una fase inicial de 11 días y una experimental de 70 días. El estudio se efectuó en la época climática de nortes (período de viento, lluvia y reducción de la temperatura ambiente).

Se utilizaron 28 corderas Pelibuey $\mathrm{x}$ Blackbelly con una edad de $89,4 \pm 2,2$ días y $13,2 \pm 0,42 \mathrm{~kg}$ de peso corporal.

Las corderas se distribuyeron al azar a uno de dos tratamientos (14 corderas por tratamiento). Se utilizó un diseño completamente al azar. El factor fue el nivel de complementación energética y proteínica: complementación alimenticia baja (CAB) vs. complementación alimenticia media (CAM). La unidad experimental fue la cordera.

Las corderas se alimentaron con base en pastoreo (9:00 a 18:00h) y complementación energética y proteínica.

Para el pastoreo, se ocupó una superficie de 0,723 ha con Cynodon plectostachyus, que se dividió en seis secciones similares (tres por tratamiento). Se utilizó un pastoreo rotacional. Las praderas se fertilizaron con urea (33 kg N ha ${ }^{-1}$ época climática $\left.{ }^{-1}\right)$.

El consumo de pasto promedio por cordera se calculó mediante la metodología del doble muestreo comparativo (Haydock y Shaw, 1975).

Para establecer el promedio de consumo de pasto por cordera se consideró el pasto desaparecido, el número de días que se ocupó en una sección y el número de corderas en pastoreo dentro de cada sección.

En cada sección se tomaron muestras de pasto (al iniciar y finalizar el pastoreo) y se determinó el porcentaje de materia seca(MS) y proteína bruta $(\mathrm{PB})$.

El estado de la pradera se evaluó a través de las variables cobertura $(\%)$, altura $(\mathrm{cm})$ y producción de materia seca $(\mathrm{kg})$. La evaluación de cada sección de pastoreo se realizó al iniciar y finalizar el pastoreo. Las diversas variables estudiadas en la pradera se midieron en un área de $0,25 \mathrm{~m}^{2}$.

Se utilizó un complemento alimenticio (2,57 Mcal EM kg-1; 16,1\% PB) elaborado con pulidura de arroz $(65 \%)$ y pasta de coco (35\%).

En CAB se ofreció 70 g cordera $^{-1} \mathrm{~d}^{-1}(0,18$ Mcal de EM cordera ${ }^{-1} \mathrm{~d}^{-1}$ y $10,5 \mathrm{~g}$ PB cordera $\left.^{-1} \mathrm{~d}^{-1}\right)$, mientras que en CAM $210 \mathrm{~g}$ cordera $^{-1} \mathrm{~d}^{-1}(0,54$ Mcal EM y $31,6 \mathrm{~g}$ PB cordera $\left.^{-1} \mathrm{~d}^{-1}\right)$, durante los 70 días de evaluación. El complemento alimenticio se ofreció de 7:00 a 9:00 h en corrales individuales.

En ambos tratamientos se ofrecieron diariamente sales minerales (Magnophoscal ${ }^{\circledR}$ ) a libre acceso durante su alojamiento.

Se determinaron los pesos corporales (al iniciar el estudio y posteriormente cada 14 días hasta la finalización del estudio, día 70) y la GDP en las corderas.

El consumo individual de $\mathrm{MS} \mathrm{d}^{-1}, \mathrm{~PB} \mathrm{~d}^{-1}$ y $\mathrm{EM} \mathrm{d}^{-1}$ aportado por el pasto, complemento alimenticio y por ambos se determinó en un período de 14 días.

Los datos correspondientes a las variables que determinan los cambios en la pradera y el consumo de minerales se analizaron mediante estadística descriptiva (me$\mathrm{dia} \pm \mathrm{EE}$ ). Los datos correspondientes a peso corporal y GDP se compararon mediante un análisis de varianza de manera transversal (SAS, 1999).

\section{RESULTADOSYDISCUSIÓN}

El promedio en el consumo diario de sal mineral fue de $7,1 \pm 0,7 \mathrm{~g}_{\text {cordera }}{ }^{-1}$.

El nivel de consumo del complemento alimenticio no influyó ( $p>0,05)$ en el peso corporal de las corderas. Los promedios en los pesos corporales de las corderas al inicio (día 0 ), días $14,28,42,56$ y 70 fueron $13,2 \pm 0,4 ; 12,7 \pm 0,4 ; 13,8 \pm 0,5 ; 14,5 \pm 0,5$; $15,2 \pm 0,5$ y $16,0 \pm 0,5 \mathrm{~kg}$, respectivamente. 


\section{CRECIMIENTO POSTDESTETE DE CORDERAS EN PASTOREO SUPLEMENTADAS}

De manera similar al peso corporal, la GDP a través del estudio no resultó afectada $(\mathrm{p}>0,05)$ por el nivel de consumo del complemento alimenticio. Los promedios en las GDP de las corderas entre el inicio y el día 14, día 15 a 28 , día 29 a 42 , día 43 a 56 y día 57 a 70 fueron $-32 \pm 10 ; 81 \pm 11 ; 48 \pm 7 ; 53 \pm 5$ y $68 \pm 5$ g, respectivamente. Durante los 70 días de evaluación, las corderas tuvieron una ganancia de peso de $3,0 \pm 0,25 \mathrm{~kg}_{\text {cordera }}{ }^{-1} \mathrm{y}$ una GDP de $43 \pm 4 \mathrm{~g}$ cordera $^{-1}$.

Las GDP observadas en este estudio son inferiores a las indicadas en corderas Pelibuey en pastoreo $\left(82 \mathrm{~g}\right.$ cordera $\left.^{-1} \mathrm{~d}^{-1}\right)$ (González et al., 2002a) y corderas Blackbelly en pastoreo con un bajo nivel de complementación alimenticia (69 a $79 \mathrm{~g}$ cordera $\left.\mathrm{d}^{-1} \mathrm{~d}^{-1}\right)$ (González et al., 2002b).

Las corderas del tratamiento $\mathrm{CAB}$ consumieron en promedio $56,5 \mathrm{~g} \mathrm{~d}^{-1} \mathrm{del}$ complemento alimenticio (base seca), lo que significa consumos calculados para PB y EM de 9, $1 \mathrm{~g}$ cordera d $^{-1}$ y $0,15 \mathrm{Mcal}_{\text {cordera d }}{ }^{-1}$. En CAM, en promedio consumieron 179,2 g $\mathrm{d}^{-1}$ del complemento alimenticio (base seca), lo que implica consumos calculados de 28,9 $\mathrm{g} \mathrm{PC} \mathrm{cordera} \mathrm{d}^{-1}$ y $0,46 \mathrm{Mcal} \mathrm{EM} \mathrm{cordera} \mathrm{d}^{-1}$.

En el tratamiento CAM, las corderas recibieron un mayor aporte de PB y EM (vía complemento alimenticio) con respecto al tratamiento CAB. Sin embargo, esta ventaja no fue suficiente para que las corderas del grupo CAM mostraran mayor GDP y peso corporal con respecto al grupo CAB. Es posible, que en este tipo de corderas, el suministro de EM y PB, vía el complemento alimenticio, deba ofrecerse en un mayor nivel al estudiado en el presente trabajo, para lograr una mayor GDP, tal como ocurre en corderos machos West African (Martínez et al., 2002).

Los valores de PB en C. plectostachyus al iniciar y finalizar el pastoreo fueron $11,6 \pm 0,45 \%$ y $10,0 \pm 0,33 \%$, respectivamente.

$\mathrm{Al}$ inicio del pastoreo, las praderas presentaron las siguientes características: cobertura $52 \pm 4 \%$ y altura del pasto $39 \pm 2,5$ $\mathrm{cm}$. Al final del pastoreo, cobertura $47 \pm 3 \%$ y altura del pasto $30,4 \pm 1,5 \mathrm{~cm}$.

El balance alimentario efectuado entre los días 29 a 42, permitió describir el consumo de MS de C. plectostachyus considerando el nivel de complementación alimenticia. Las corderas en $\mathrm{CAB}$ consumieron 93,7 g de pasto (en base seca) cordera ${ }^{-1} \mathrm{~d}^{-1}$, mientras que en CAM 87,9 g de pasto cordera $^{-1} \mathrm{~d}^{-1}$. Se calculó que en CAM las corderas tuvieron un consumo total de MS $(267 \mathrm{~g}$ cordera $\left.^{-1} \mathrm{~d}^{-1}\right), \operatorname{EM}\left(0,62 \mathrm{Mcal}_{\text {cordera }} \mathrm{d}^{-1}\right) \mathrm{y}$ PB (37 g cordera $\left.^{-1} \mathrm{~d}^{-1}\right)$ mayor con respecto a CAB (150 g de MS cordera ${ }^{-1} \mathrm{~d}^{-1}$; 0,32 Mcal de EM cordera ${ }^{-1} \mathrm{~d}^{-1} ; 21 \mathrm{~g}$ de $P B$ cordera $\left.^{-1} \mathrm{~d}^{-1}\right)$. Sin embargo, esta diferencia no permitió que este grupo lograra una mayor GDP $(p>0,05)$ con respecto a CAB.

En el presente estudio, las estimaciones en el consumo de MS y EM resultaron inferiores a las que señala Castellanos (1989) para corderos de $15 \mathrm{~kg}$ que ganan $50 \mathrm{~g} \mathrm{~d}^{-1}$ $\left(802 \mathrm{~g} \mathrm{MS} \mathrm{d}^{-1} ; 1,7 \mathrm{Mcal} \mathrm{EM} \mathrm{cordero} \mathrm{d}^{-1}\right)$. Lo anterior explica, en parte, la baja eficiencia de crecimiento que mostraron las corderas estudiadas.

Las corderas mostraron un bajo consumo de pasto durante el pastoreo y no se detectaron diferencias marcadas en los niveles de consumo de MS y PB entre CAB y CAM. No obstante, las variables que definieron el estado de la pradera, al iniciar y finalizar el pastoreo, indican que las corderas siempre dispusieron de pastura durante los períodos estudiados.

La baja GDP detectada en este tipo de corderas, sugiere considerar la posible participación de otros factores. Por ejemplo, la influencia de factores de tipo climático en la GDP de los animales en pastoreo (MoraMorelos et al., 2005). Al respecto, Hassen et al. (2002) indican que la época climática tiene influencia en el peso corporal postdestete de los corderos nativos y cruzados (razas ovinas de Etiopía), y señalan que durante la seca (época con reducida disponibilidad de pastura) se obtienen los menores pesos en los corderos $(11,9 \mathrm{~kg}$ a los 120 días) con respecto a los pesos registra- 
dos en los corderos durante la época con lluvias moderadas (13,0 kg a los 120 días) e intensas (12,7 $\mathrm{kg}$ a los 120 días).

Adicionalmente, Krysl y Hess (1993) en una revisión efectuada sobre comportamiento de bovinos en pastoreo, indican que la temperatura ambiente, velocidad del viento y presión barométrica alteran las actividades de pastoreo de los bovinos durante el invierno. El presente trabajo, se realizó durante la época de nortes, por lo que es factible que la cantidad de precipitación pluvial, el número de días con lluvia y la duración de la misma, hayan ejercido una influencia negativa en el crecimiento de las corderas, debido a que durante los períodos de lluvia, los corderos no pastorean.

\section{BIBLIOGRAFÍA}

Anónimo. 2007. Anuario estadístico Tabasco. Instituto Nacional de Estadística Geografía e Informática. Gobierno del Estado de Tabasco. 573 pp.

Castellanos, R.A.F. 1989. Requerimientos alimenticios del borrego Pelibuey. En: Castellanos, R.A.F. y C. Arellano (eds). Tecnologías para la producción de ovejas tropicales. Instituto $\mathrm{Na}$ cional de Investigaciones Forestales y Agropecuarias. Organización de las Naciones Unidas para la Agricultura y Alimentación. Yucatán. México. pp. 78-90.

González, R.A., Martínez, B.I., Chávez, F.J.A. Loya, H.F.M. y Lucero, M.F.A. 2002a. Crecimiento de corderos Pelibuey en pastoreo. En: memorias del II Taller Ovino del Golfo y Noreste de México. Universidad Autónoma de Tamaulipas. Cd. Victoria. Tamaulipas. México. pp. 17-20.

González, G.R., Torres, G. y Castillo, M.A. 2002b. Crecimiento de corderos Blackbelly entre el nacimiento y el peso final en el trópico húmedo de México. Vet. Méx., 33: 443-453.

Haydock, K.P. and Shaw, N.H. 1975. The comparative yield method for estimating dry matter yield of pasture. Aust. J. Exp. Agri. Anim.
Adicionalmente, si los suelos se inundan, se reducen las áreas susceptibles de ser pastoreadas por los corderos.

Se concluye que en corderas Pelibuey $\mathrm{x}$ Blackbelly en pastoreo, un nivel de complementación alimenticia medio en energía y proteína cruda no incrementa el peso corporal ni la ganancia diaria de peso con respecto a un nivel de complementación bajo. En ambos tratamientos, complementación alimenticia medio y baja, las corderas mostraron un bajo consumo de materia seca y proteína bruta durante el pastoreo.

\section{AGRADECIMIENTOS}

El presente material forma parte del proyecto 16449 FOMIX Tabasco 2005-1.

Husb., 15: 663-670.

Hassen, Y., Sölkner, J., Gizaw, S. and Baumung, R. 2002. Performance of crossbred and indigenous sheep under village condition in the cool highlands of central-northern Ethiopia: growth, birth and body weights. Small Ruminant Res., 43: 195-202.

Krysl, L.J. and Hess, B.W. 1993. Influence of supplementation on behavior of grazing cattle. J. Anim. Sci., 71: 2546-2555.

Martínez, A.M., Bravo, J., Betancourt, M., Bracho, I. y Quintana, H. 2002. Influencia de la suplementación proteica sobre el crecimiento de corderos postdestete. Zootecnia Trop., 20: 307-317.

Mora-Morelos, H., Hinojosa-Cuéllar, J.A. y OlivaHernández, J. 2005. Ganancia de peso de los corderos Pelibuey en pastoreo y con complemento alimenticio. Tecnociencia Universitaria, 10: 21-30.

Oliva, H.J. y Vidal, A. 2001. Utilización del zeranol en borregos Pelibuey en pastoreo y con concentrado energético. Universidad y Ciencia, 17: 57-64.

SAS. 1999. Statistical Analysis System. Users Guide. SAS Institute. Cary. N.C. USA. 